\title{
Quercetin educes hydroxyurea induced cytotoxicity in Immortalized mouse aortic endothelial cells
}

\author{
Zachary Kiser ${ }^{\text {Corresp., }}{ }^{1}$, Monica McGee ${ }^{2}$, Racquel Wright ${ }^{3}$, Alexander Quarshie ${ }^{4}$, Gale W Newman ${ }^{1}$, Karen \\ Randall $^{5}$, Jonathan K Stiles ${ }^{1}$, Adel Driss ${ }^{6}$, Jacqueline M Hibbert ${ }^{\text {Corresp. } 1}$ \\ ${ }^{1}$ Department of Microbiology, Biochemistry, and Immunology, Morehouse School of Medicine, Atlanta, Georgia, United States \\ 2 Spelman College, Atlanta, Georgia, United States \\ 3 Biotechnology Centre, University of the West Indies, Mona, Mona, Jamaica \\ 4 Community Health \& Preventive Medicine, Morehouse School of Medicine, Atlanta, Georgia, United States \\ 5 Department of Pharmacology \& Toxicology, Morehouse School of Medicine, Atlanta, Georgia, United States \\ 6 Department of Physiology, Morehouse School of Medicine, Atlanta, Georgia, United States \\ Corresponding Authors: Zachary Kiser, Jacqueline M Hibbert \\ Email address: zkiser@msm.edu, jhibbert@msm.edu
}

Background Chronic inflammation is a characteristic of sickle cell disease (SCD), and is invariably associated with vascular endothelial injury. Hydroxyurea (HU), a naturally cytotoxic chemotherapeutic agent, is the only FDA drug approved for SCD, and is therefore naturally cytotoxic. Quercetin (QCT) is a dietary flavonoid found ubiquitously in plants and foods that have anti-oxidative and anti-inflammatory characteristics. Our hypothesis is that dietary QCT will decrease cytotoxic effects of lipopolysaccharide (LPS) and HU induced vascular cell damage. LPS was used to induce an inflammatory response in immortalized mouse aortic endothelial cells (iMAECs), representing an in vitro model of inflamed vascular endothelium.

Methods Lipopolysaccharide (LPS) was used to induce inflammation in immortalized mouse aortic endothelial cells (iMAECs), providing an in vitro model of inflamed endothelial cells. The cells were exposed to LPS throughout the entire experiment. Interventions included treating the LPS exposed cells with QCT, HU, or QCT + HU over 50 hours. The 50 -hour period included 24 hours of varying treatments, followed by two hours of hypoxic exposure and then 24 hours under normal aerobic exposure.

Results LDH level was significantly higher for LPS treated versus untreated cells $(P=0.0004)$. LPS plus 30 micromole QCT reduced the LDH ( $p=0.1$, trend), whereas LPS plus 100 micromoles HU, significantly increased LDH $(p=0.0004)$. However, LPS plus treatment with 30 micromoles QCT/100 micromoles HU, significantly reduced $L D H$, compared with HU alone $(p=0.0002)$.

Discussion These results suggest that quercetin may be effective against vascular endothelial cell damage for iMAECs in vitro. In particular, it shows promise in preventing HU-induced cytotoxicity, surprisingly found from these results. This latter finding is interesting, and should be given more consideration, since $\mathrm{HU}$ is the only FDA-approved drug for treating sickle cell patients, and its use is rapidly increasing. 
1

2 Quercetin Reduces Hydroxyurea Induced Cytotoxicity in Immortalized Mouse Aortic

3 Endothelial Cells

4 Zachary M. Kiser, ${ }^{1}$ Monica DM. McGee, ${ }^{2}$ Racquel J. Wright ${ }^{3}$ Alexander Quarshie, ${ }^{1}$ Gale W.

5 Newman, ${ }^{1}$ Karen R. Randall, ${ }^{1}$ Jonathan K. Stiles, ${ }^{1}$ Adel Driss,,${ }^{1}$ Jacqueline M. Hibbert ${ }^{1}$

$6{ }^{1}$ Morehouse School of Medicine, 720 Westview Dr SW, Atlanta, GA 30303 USA

$7 \quad 2$ Spelman College, 350 Spelman Ln, Atlanta, GA 30314 USA

$8{ }^{3}$ University of the West Indies Mona, Kingston 7 Jamaica

9

10 Corresponding Author:

11 Jacqueline M. Hibbert ${ }^{1}$

12 Morehouse School of Medicine, 720 Westview Dr SW, Atlanta, GA 30303 USA

13 Email address: jhibbert@msm.edu 
15

16

17

18

19

20

21

22

23

24

25

26

27

28

29

30

31

32

33

34 35

36

37

38 39

40

41

42

43

Abstract

\section{Background}

Chronic inflammation is a characteristic of sickle cell disease (SCD), and is invariably associated with vascular endothelial injury. Hydroxyurea (HU), a naturally cytotoxic chemotherapeutic agent, is the only FDA drug approved for SCD, and is therefore naturally cytotoxic. Quercetin (QCT) is a dietary flavonoid found ubiquitously in plants and foods that have anti-oxidative and anti-inflammatory characteristics. Our hypothesis is that dietary QCT will decrease cytotoxic effects of lipopolysaccharide (LPS) and HU induced vascular cell damage. LPS was used to induce an inflammatory response in immortalized mouse aortic endothelial cells (iMAECs), representing an in vitro model of inflamed vascular endothelium.

\section{Methods}

Lipopolysaccharide (LPS) was used to induce inflammation in immortalized mouse aortic endothelial cells (iMAECs), providing an in vitro model of inflamed endothelial cells. The cells were exposed to LPS throughout the entire experiment. Interventions included treating the LPS exposed cells with QCT, HU, or QCT + HU over 50 hours. The 50-hour period included 24 hours of varying treatments, followed by two hours of hypoxic exposure and then 24 hours under normal aerobic exposure.

\section{Results}

8 LDH level was significantly higher for LPS treated versus untreated cells $(P=0.0004)$. LPS plus 930 micromole QCT reduced the LDH ( $\mathrm{p}=0.1$, trend), whereas LPS plus 100 micromoles HU, 0 significantly increased LDH $(\mathrm{p}=0.0004)$. However, LPS plus treatment with 30 micromoles 1 QCT/100 micromoles HU, significantly reduced LDH, compared with HU alone $(\mathrm{p}=0.0002)$.

\section{Discussion}

3 These results suggest that quercetin may be effective against vascular endothelial cell damage for 
44 iMAECs in vitro. In particular, it shows promise in preventing HU-induced cytotoxicity, 45 surprisingly found from these results. This latter finding is interesting, and should be given more 46

47 use is rapidly increasing.
49

50

51 52 53 54 55 56 57 58 59

65

\section{$\underline{\text { Introduction }}$}

Sickle Cell Disease (SCD) is a genetic disease caused by a single nucleotide mutation in the beta globin gene, which increases the tendency of hemoglobin to polymerize and causes red blood cells (RBC) to acquire a sickle shape (Pauling \& Itano, 1949). Accumulation of the polymerized hemoglobin $\mathrm{S}(\mathrm{HbS})$ within the RBC causes injury to the red cell, altering both its form and function. Accumulation of the damaged red blood cells within the blood vessels causes decreased blood flow and hence, low oxygen delivery. The low oxygen levels promote more red cell deformation, and increased polymerization in the already damaged red cells (Steinberg, 2008). As these damaged cells traverse the circulatory system they encounter and injure vascular endothelial cells. Hydroxyurea (HU) is the only FDA approved drug for SCD. It is a chemotherapy drug approved for use at low dose in SCD patients to increase the production of fetal $\mathrm{Hb}$, which does not polymerize, therefore maintaining normal red cell function and life span and reducing the damage caused by the sickle cells. It is important to note that the cytotoxic nature of the drug, may present a problem for long term use as an SCD treatment (Brun et al., 2003; Baz et al., 2012).

Vascular endothelial cells serve multiple physiological functions in normal individuals. These include control of vascular tone, separating blood from the interstitial spaces and the presence of molecules and receptors involved in vascular adhesion, in both health and disease (Hebbel, Osarogiagbon \& Kaul, 2004; Rajendran et al., 2013). Chronic inflammation in sickle cell patients is invariably associated with injury to the vascular endothelium. In SCD this damage leads to continuously varying activated states of vascular endothelial cells. System-wide inflammation accompanies endothelial cell activation. Increased adhesion of leukocytes to the vascular endothelium demonstrates evidence of this inflammatory state and is associated with 
74 shortening of the white cell's life span, and acquisition of a pro-adhesive phenotype by the 75 vascular endothelial cells. Oxidative stress and reactive oxygen species also develop in the 76 vascular endothelium (Hebbel, Osarogiagbon \& Kaul, 2004; Rajendran et al., 2013). The effects

77 of activation and inflammation lead to further complications within the endothelial cells, which

78 include increased levels of inflammatory cytokines and adhesion molecules, hypoxia-reperfusion 79 injury, increased reactive oxygen production and possible vaso-occlusion (Osarogiagbon et al., 80 2000; Koo et al., 2001; Hebbel, Osarogiagbon \& Kaul, 2004; Cook-Mills, Marchese \& Abdala81 Valencia, 2011; Chirico \& Pialoux, 2012).

82

83 Ischemia/reperfusion injury (IRI) occurs when the blood supply returns to tissue that was

84 previously subjected to ischemia. Reflux of oxygen and nutrients to the formerly ischemic tissue 85 destroys cellular DNA, specific proteins and the plasma membrane. The damage related to IRI 86 stems from oxidative stress, which causes a non-specific inflammatory state in the vasculature. Ischemia/reperfusion injury also induces the production of oxygen free radicals. The increased production of oxygen free radicals causes the formation of reactive oxygen species. The rapid buildup of free radicals during the reperfusion phase of IRI causes the body's natural sources of antioxidants, such as superoxide dismutase and glutathione, to be depleted during the period of ischemia (Koo et al., 2001). The depletion of natural antioxidants allows oxidative damage to progress unchecked causing further injury to the endothelium. A supplemental antioxidant supply, such as may be obtained from the flavonoid quercetin (QCT), may help to prevent the increasing free radical production (Hyacinth, Gee \& Hibbert, 2010) as well as the damage to macromolecules, including DNA (Potenza et al., 2008).

The literature describes quercetin as a reliable anti-oxidant for in vitro studies (Potenza et al., 2008; Boots, Haenen \& Bast, 2008; Dajas et al., 2015; D’Andrea, 2015; Sharma et al., 2015).

The enzyme Xanthine Oxidase is one source of reactive oxygen species production (Kelley et al., 2010; Cantu-Medellin \& Kelley, 2013) and reports in the literature show that QCT is an effective inhibitor of xanthine oxidase (Middleton, Kandaswami \& Theoharides, 2000; Pauff \& Hille, 2009; Cao, Pauff \& Hille, 2014). The use of QCT as a supplemental source of antioxidants may help to reduce the drain on the already weak anti-oxidant levels in SCD patients. Based on the antioxidant properties of QCT, in the present study we investigated whether QCT 
105 may decrease the level of cytotoxicity associated with HU treatment on an inflammation/hypoxia 106 model.

107

108

109

110

111

112

113

114

115

116

117

118

119

120

121

122

123

124

125

126

127

128

129

130

131

132

133

134

\section{Materials and Methods}

\section{Culture of Immortalized Mouse Aortic Endothelial Cells}

Immortalized Mouse Aortic Endothelial Cells (iMAEC), a stable cell line derived from endothelium of the mouse aorta, were obtained through a generous gift from the Jo Lab at Emory University (Atlanta, GA) (Ni et al., 2014). Cells were grown in Dulbecco’s Modified Eagle Medium (DMEM) containing 10\% fetal bovine serum, 1\% 100X MEM Non-Essential Amino Acids Solution (MNEAA, Gibco, Waltham, MA), 1.3\% Endothelial Cell Growth Supplement (ECGS) (Gibco, Waltham, MA, final concentration $50 \mu \mathrm{g} / \mathrm{ml}$ ) and 1\% 100X penicillinstreptomycin, following previously published procedures (Koo et al., 2001). The cultures were kept in a humidified chamber at $5 \% \mathrm{CO}_{2}$ and $37^{\circ} \mathrm{C}$, and the medium was changed every 1 to 2 days.

\section{Treatment of Immortalized Mouse Aortic Endothelial Cells}

iMAEC were seeded at a density of 10,000 cells/well and allowed to grow to approximately $70 \%$ confluency, which occurred in approximately 36 hours. After the desired confluence was achieved, the iMAEC were treated with $250 \mathrm{ng} / \mathrm{ml}$ of lipopolysaccharide (Sigma-Aldrich, St.

Louis, MO) along with varying concentrations of Quercetin (M.W. $302.24 \mathrm{~g} / \mathrm{mol}$ ) (QCT) (Sigma-Aldrich, St. Louis, MO), Hydroxyurea (M.W. 76.0547 g/mol) (HU) (Sigma-Aldrich, St. Louis, MO) or a combination of Quercetin + Hydroxyurea (QCT+HU). The treatment with QCT, HU, or QCT + HU, lasted for 24 hours. The cells were then subjected to two hours of enzymatic hypoxia, which is a model for ischemia-reperfusion injury, achieved using a combination glucose oxidase (GOX) (Sigma-Aldrich, St. Louis, MO) and catalase (CAT) (Sigma-Aldrich, St. Louis, MO), both from Sigma-Aldrich, as previously described (Mueller, Millonig \& Waite, 2009). Briefly, the GOX and CAT enzymes were added to high glucose 
135 DMEM (4.5 g/L glucose; Thermo-Fisher, Waltham, MA) and the resulting mixture placed on the 136 confluent iMAEC for two hours of incubation. Hypoxia was subsequently terminated by

137 removing the GOX/CAT DMEM and replacing it with normal DMEM containing $250 \mathrm{ng} / \mathrm{ml}$ of

138 LPS. The iMAEC were then allowed to incubate in the normal DMEM/LPS media for 24 hours 139 after which cytotoxicity was measured. (See Figure 1 for outline of experiment)

140

141

142

143

144

145

146

147

148

149

150

151

152

153

154

155

156

157 158

159

160

161

162

163

164

165

\section{Figure 1}

Measurement of Cytotoxicity

LDH released from the cells into the medium, was measured as per the manufacturer's protocol (Pierce LDH Cytotoxicity Assay; ThermoFisher, Waltham, MA), and served as a biomarker for cellular cytotoxicity following the cell treatments. Before starting the assay, $10 \mu 1$ of $10 \mathrm{X}$ lysis buffer was added to healthy iMAEC cells and allowed to incubate at $37^{\circ}$ for 45 minutes. These cells served as the positive control, indicating the expected mean concentration of LDH in each well. After 24 hours of normal aerobic exposure, $50 \mu$ of supernatant from each well was transferred to a new 96-well plate, $50 \mu \mathrm{l}$ of substrate solution was added per the manufacturer's protocol and the cells were incubated at room temperature for 30 minutes. After 30 minutes, $50 \mu 1$ of stop solution was added to each well. The absorbance for each well was then measured by spectrophotometer at 490nm and $680 \mathrm{~nm}$ to account for background absorbance. To quantify LDH release, the absorbance of each treatment group was divided by the absorbance of the positive control group and multiplied by 100 . Each result was expressed as a percentage of total LDH release.

\section{Statistical Analysis}

Results are presented as means $\pm \mathrm{SD}$. The D'Agostino's $\mathrm{K}^{2}$ test was used to measure the distribution of the data. The data was not normally distributed; hence the Mann-Whitney U Test was used to compare differences by treatment. Based on the results of the D'Agostino's $\mathrm{K}^{2}$ test, the Kruskal-Wallis non-parametric analysis of variance with pair-wise comparison was utilized. 
166 Statistical significance was assumed at $\mathrm{p} \leq 0.05$ for all analyses. No outliers, designated as $\mathrm{SD} \pm 2$, 167 were identified. The data were analyzed using GraphPad Prism 7 for MacOS Sierra.

168

169 Results

170

171 To establish whether LPS (Inflammatory Model, $250 \mathrm{ng} / \mathrm{ml}$ ) causes increased LDH release, two

172 groups of cells were compared. The mean absorbance of the LPS treated cells was significantly

173 higher than for the untreated cells $(0.60 \pm 0.17$ vs $0.42 \pm 0.12, \mathrm{p}=0.0004)$ (Figure 2$)$. After

174 confirming that LPS causes increased LDH release, we sought to determine whether various

175 concentrations of QCT could prevent LDH release. The percentage of LDH released from the

$17630 \mu \mathrm{mol}$ QCT-treated cells trended lower than the LDH\% measured in the inflammatory model

177 (LPS, $250 \mathrm{ng} / \mathrm{ml})(\mathrm{p}=0.1$, trend) (Figure 3). Those cells treated with $100 \mu \mathrm{mol}$ of HU, showed a

178 significantly higher percentage of LDH release compared with LPS inflammation alone (14.4 \pm

1796.87 vs. $5.67 \pm 5.73, \mathrm{p}=0.0004)$. The addition of $30 \mu \mathrm{mol}$ of QCT with the HU treatment gleaned

180 a significant reduction of LDH release, compared with only HU treatment (5.55 \pm 4.66 vs. $14.4 \pm$

181 6.87, $\mathrm{p}=0.0002$ ). Interestingly, the combination treatment (QCT 30/100 $\mu \mathrm{mol} \mathrm{HU}$ ) returned LDH

182 levels to those of the baseline LPS treated group (250 ng/ml), (Figure 4).

183

184

185

186

187

188

189

190

191

192

193

194 195

\section{Figure 2}

Figure 3

Figure 4

\section{$\underline{\text { Discussion and Conclusions }}$}

Damage to vascular endothelial cells in SCD patients contributes considerably to the progression and severity of the disease (Hebbel, Osarogiagbon \& Kaul, 2004; Kaul, Finnegan \& Barabino, 2009). One form of injury to the endothelial cells results from cyclical periods of ischemia and 
196 reperfusion which exacerbate the endothelial cell damage and contribute to the progression of the 197 disease (Hebbel, 2014). Since endothelial cells are a primary site of pathogenesis, there is

198

199

200

201

202

203

204

205

206

207

208

209

210

211

212

213

214

215

216

217

218

219

220

221

222

223

224

225

226

considerable interest in developing treatments that target inflammatory mechanisms in these cells. This study was designed to determine if QCT, a plant flavonoid with anti-oxidant and antiinflammatory properties, could reduce or prevent the damage caused by inflammation/hypoxia and damage caused by HU. Reports in the literature indicate that LDH is both a general marker of cellular damage and a marker of ischemia-reperfusion damage in SCD (Stankovic Stojanovic \& Lionnet, 2016). This study used LDH as a marker for assessing the efficacy of QCT treatment for reducing inflammation in an in-vitro model. The first result confirmed that QCT (30 $\mu \mathrm{mol})$ treatment showed a trend toward reducing the percentage of LDH released by an LPS induced inflammatory model undergoing a period of hypoxia and re-oxygenation. This lends preliminary support to the idea that QCT could be useful for reducing cytotoxicity caused by inflammation and oxidative stress in endothelial cells. The second finding that $\mathrm{HU}(100 \mu \mathrm{mol})$, significantly increased the amount of LDH released in the inflammatory model, during a period of hypoxia and re-oxygenation, is novel since to our knowledge, there are no reports in the literature about this effect of low dose HU for treating SCD (Brun et al., 2003; Baz et al., 2012). By reducing the damage in endothelial cells, a key site of disease progression in SCD (Osarogiagbon et al., 2000; Hebbel, Osarogiagbon \& Kaul, 2004; Rajendran et al., 2013; Hebbel, 2014), more severe disease side effects such as atherosclerosis (Elsharawy, Moghazy \& Shawarby, 2009), future clot formation, and vessel stenosis (Adams, 2007; Doepp et al., 2012; Sparkenbaugh \& Pawlinski, 2013) could be alleviated or completely prevented. However, any reduction of baseline inflammation related to QCT treatment, could be reversed by subsequent inflammatory response.

While QCT may not completely avert damage caused by the combined effect of inflammation, oxidative stress and HU treatment, these findings suggest that the QCT may prevent injury from the HU drug, shown in these studies to cause inflammation, but which has previously been assumed only to reduce the subclinical ischemic events induced by sickle red blood cells. The combination of QCT and $\mathrm{HU}$, resulted in significant reduction of LDH release to baseline levels of inflammation, indicating that QCT may completely prevent damage induced by HU treatment, possibly returning the endothelial cells to pre-treatment levels. Therefore, a combination treatment of QCT with the HU, may prevent or reduce HU treatment induced endothelial cell 
227 damage. Suggested QCT use as adjunct to HU therapy may translate to improved disease 228 outcomes.

229

230 This study was limited to investigating only one type of endothelial cell, aortic endothelial cells.

231 While there is vast homogeneity among endothelial cell types there are still subtle differences

232 that may affect the outcome of QCT treatment (Aird, 2012). It is a vital future step to repeat

233 these experiments in endothelial cells from several different locations in the body and of human

234 origin. This could confirm QCT efficacy system wide. Once confirmed in vitro then in vivo

235 models will need to be tested.

236

237 In summary, this study demonstrates a new and important finding that HU treatment could be 238 increasing inflammation in the already chronically inflamed vascular endothelium in patients 239 with SCD. The study successfully demonstrated that QCT may be an effective treatment in 240 reducing cytotoxicity caused by inflammation resulting from ischemia-reperfusion, and by 241 treatment with HU. Given that HU is an important pharmacological agent used in the treatment 242 of SCD, it may advantageous to reduce possible short and long term cytotoxic effects associated 243 with HU treatment. These studies show that pre-treatment with QCT may provide protection 244 from endothelial cell damage caused by inflammation and hypoxic periods. Use of QCT as an 245 adjunct treatment with HU could reduce the amount of HU required, limit endothelial cell injury 246 and possibly decrease the severity of secondary disease consequences, such as stroke, vessel 247 stenosis, and vasculopathy. Based on the results of this study, adding QCT to the standard 248 treatment regimen should be seriously considered.

249

\section{Acknowledgements}

251 We would like to give our gratitude to Dr. Sharon Francis for her guidance and support during 252 these experiments. We also appreciate the members of Jo Lab at Emory University for their gift 253 of the iMAEC and technical guidance.

254

255

256

257

$\underline{\text { References }}$ 
258

259

260

261

262

263

264

265

266

267

268

269

270

271

272

273

274

275

276

277

278

279

Adams RJ. 2007. Big strokes in small persons. Archives of Neurology 64:1567-1574. DOI: 10.1001/archneur.64.11.1567.

Aird WC. 2012. Endothelial Cell Heterogeneity. Cold Spring Harbor Perspectives in Medicine 2. DOI: 10.1101/cshperspect.a006429.

Baz W., Najfeld V., Yotsuya M., Talwar J., Terjanian T., Forte F. 2012. Development of myelodysplastic syndrome and acute myeloid leukemia 15 years after hydroxyurea use in a patient with sickle cell anemia. Clinical Medicine Insights. Oncology 6:149-152. DOI: 10.4137/CMO.S8810.

Bindoli A., Valente M., Cavallini L. 1985. Inhibitory action of quercetin on xanthine oxidase and xanthine dehydrogenase activity. Pharmacological Research Communications 17:831839.

Boots AW., Haenen GRMM., Bast A. 2008. Health effects of quercetin: from antioxidant to nutraceutical. European Journal of Pharmacology 585:325-337. DOI: 10.1016/j.ejphar.2008.03.008.

Brun M., Bourdoulous S., Couraud PO., Elion J., Krishnamoorthy R., Lapoumeroulie C. 2003. Hydroxyurea downregulates endothelin-1 gene expression and upregulates ICAM-1 gene expression in cultured human endothelial cells. The Pharmacogenomics Journal 3:215-226. DOI: 10.1038/sj.tpj.6500176.

Cantu-Medellin N., Kelley EE. 2013. Xanthine oxidoreductase-catalyzed reactive species generation: A process in critical need of reevaluation. Redox Biology 1:353-358. DOI: 10.1016/j.redox.2013.05.002. 
280 Cao H., Pauff JM., Hille R. 2014. X-ray Crystal Structure of a Xanthine Oxidase Complex with the 281 Flavonoid Inhibitor Quercetin. Journal of Natural Products 77:1693-1699. DOI:

282 10.1021/np500320g.

283

284

285

286

287

288

289

290

291

292

293

294

295

296

297

298

299

300

301

IUBMB life 64:72-80. DOI: 10.1002/iub.584.

Cook-Mills JM., Marchese ME., Abdala-Valencia H. 2011. Vascular cell adhesion molecule-1 expression and signaling during disease: regulation by reactive oxygen species and antioxidants. Antioxidants \& Redox Signaling 15:1607-1638. DOI:

10.1089/ars.2010.3522.

Dajas F., Abin-Carriquiry JA., Arredondo F., Blasina F., Echeverry C., Martínez M., Rivera F., Vaamonde L. 2015. Quercetin in brain diseases: Potential and limits. Neurochemistry International 89:140-148. DOI: 10.1016/j.neuint.2015.07.002.

D'Andrea G. 2015. Quercetin: A flavonol with multifaceted therapeutic applications? Fitoterapia 106:256-271. DOI: 10.1016/j.fitote.2015.09.018.

Doepp F., Kebelmann-Betzing C., Kivi A., Schreiber SJ. 2012. Stenosis or hyperperfusion in sickle cell disease--ultrasound assessment of cerebral blood flow volume. Ultrasound in Medicine \& Biology 38:1333-1338. DOI: 10.1016/j.ultrasmedbio.2012.04.003.

Elsharawy MA., Moghazy KM., Shawarby MA. 2009. Atherosclerosis in sickle cell disease - a review. The International Journal of Angiology : Official Publication of the International College of Angiology, Inc 18:62-66.

Hebbel RP. 2014. Ischemia-reperfusion injury in sickle cell anemia: relationship to acute chest syndrome, endothelial dysfunction, arterial vasculopathy, and inflammatory pain. 
302

303

304

305

306

307

308

309

310

311

312

313

314

315

316

317

318

319

320

321

322

323

Hematology/Oncology Clinics of North America 28:181-198. DOI:

10.1016/j.hoc.2013.11.005.

Hebbel RP., Osarogiagbon R., Kaul D. 2004. The endothelial biology of sickle cell disease: inflammation and a chronic vasculopathy. Microcirculation (New York, N.Y.: 1994) 11:129-151.

Hyacinth HI., Gee BE., Hibbert JM. 2010. The Role of Nutrition in Sickle Cell Disease. Nutrition and Metabolic Insights 3:57-67. DOI: 10.4137/NMI.S5048.

Kaul DK., Finnegan E., Barabino GA. 2009. Sickle Red Cell - Endothelium Interactions. Microcirculation (New York, N.Y. : 1994) 16:97-111. DOI: 10.1080/10739680802279394.

Kelley EE., Khoo NKH., Hundley NJ., Malik UZ., Freeman BA., Tarpey MM. 2010. Hydrogen peroxide is the major oxidant product of xanthine oxidase. Free Radical Biology \& Medicine 48:493-498. DOI: 10.1016/j.freeradbiomed.2009.11.012.

Koo DD., Welsh KI., West NE., Channon KM., Penington AJ., Roake JA., Morris PJ., Fuggle SV. 2001. Endothelial cell protection against ischemia/reperfusion injury by lecithinized superoxide dismutase. Kidney International 60:786-796. DOI: 10.1046/j.15231755.2001.060002786.x.

Middleton E., Kandaswami C., Theoharides TC. 2000. The Effects of Plant Flavonoids on Mammalian Cells:Implications for Inflammation, Heart Disease, and Cancer. Pharmacological Reviews 52:673-751.

Mueller S., Millonig G., Waite GN. 2009. The GOX/CAT system: a novel enzymatic method to independently control hydrogen peroxide and hypoxia in cell culture. Advances in Medical Sciences 54:121-135. DOI: 10.2478/v10039-009-0042-3. 
324 Ni C-W., Kumar S., Ankeny CJ., Jo H. 2014. Development of immortalized mouse aortic 325 endothelial cell lines. Vascular Cell 6:7. DOI: 10.1186/2045-824X-6-7.

326

327

328

329

330

331

332

333

Osarogiagbon UR., Choong S., Belcher JD., Vercellotti GM., Paller MS., Hebbel RP. 2000. Reperfusion injury pathophysiology in sickle transgenic mice. Blood 96:314-320.

Pauff JM., Hille R. 2009. Inhibition Studies of Bovine Xanthine Oxidase by Luteolin, Silibinin, Quercetin, and Curcumin. Journal of natural products 72:725-731. DOI: 10.1021/np8007123.

Pauling L., Itano HA. 1949. Sickle cell anemia a molecular disease. Science (New York, N.Y.) 110:543-548.

Potenza L., Calcabrini C., Bellis RD., Mancini U., Cucchiarini L., Dachà M. 2008. Effect of quercetin on oxidative nuclear and mitochondrial Dna damage. Biofactors 33:33-48.

Rajendran P., Rengarajan T., Thangavel J., Nishigaki Y., Sakthisekaran D., Sethi G., Nishigaki I. 2013. The Vascular Endothelium and Human Diseases. International Journal of Biological Sciences 9:1057-1069. DOI: 10.7150/ijbs.7502.

Sharma DR., Sunkaria A., Wani WY., Sharma RK., Verma D., Priyanka K., Bal A., Gill KD. 2015. Quercetin protects against aluminium induced oxidative stress and promotes mitochondrial biogenesis via activation of the PGC-1 $\alpha$ signaling pathway. NeuroToxicology 51:116-137. DOI: 10.1016/j.neuro.2015.10.002.

Sparkenbaugh E., Pawlinski R. 2013. Interplay between coagulation and vascular inflammation in sickle cell disease. British journal of haematology 162. DOI: 10.1111/bjh.12336. 
344 Stankovic Stojanovic K., Lionnet F. 2016. Lactate dehydrogenase in sickle cell disease. Clinica 345 Chimica Acta; International Journal of Clinical Chemistry 458:99-102. DOI: 10.1016/j.cca.2016.04.035.

347 Steinberg MH. 2008. Sickle cell anemia, the first molecular disease: overview of molecular 348 etiology, pathophysiology, and therapeutic approaches. TheScientificWorldJournal 349 8:1295-1324. DOI: 10.1100/tsw.2008.157.

350

351 
Figure 1

Experimental Outline

Timeline of Experimental Procedure
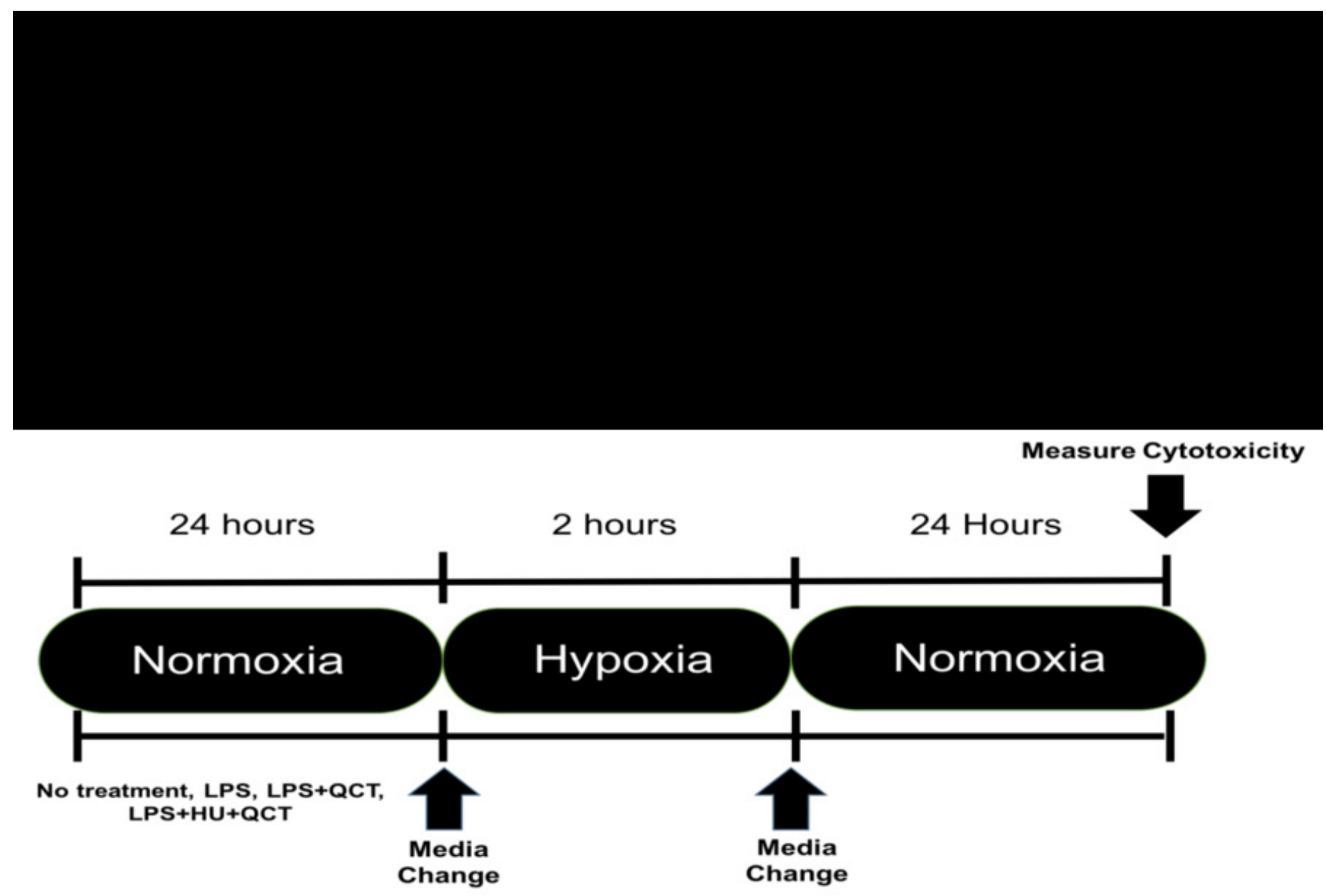
Figure 2

Comparing LDH release in LPS treated cells versus non-treated cells.

Values are means +/- SD. The mean LDH release of the LPS treated cells was significantly higher than for the untreated cells.

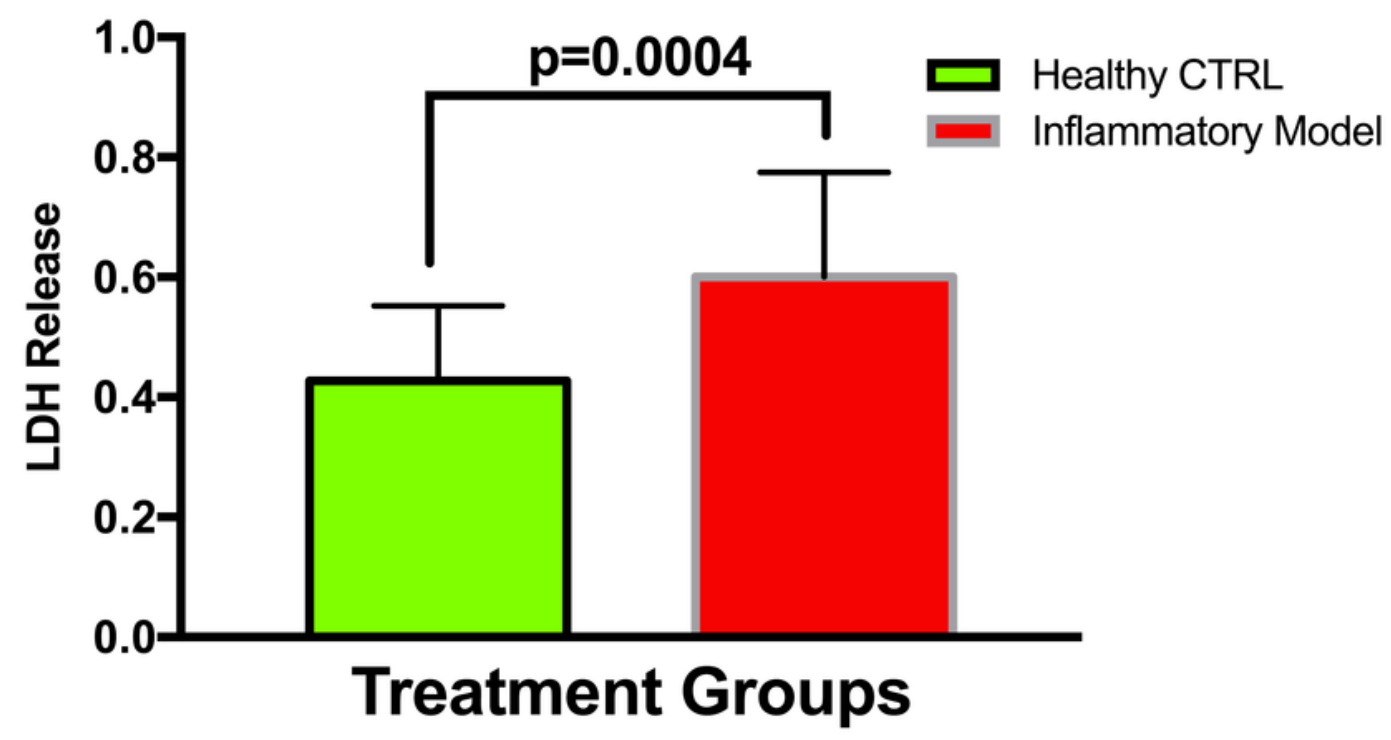


Figure 3

The effect of various concentrations of quer on LDH release.

Values are means +/- SD. The percentage of LDH released from the $30 \mu \mathrm{mol}$ QCT-treated cells trended lower than the LDH release measured in the inflammatory model.

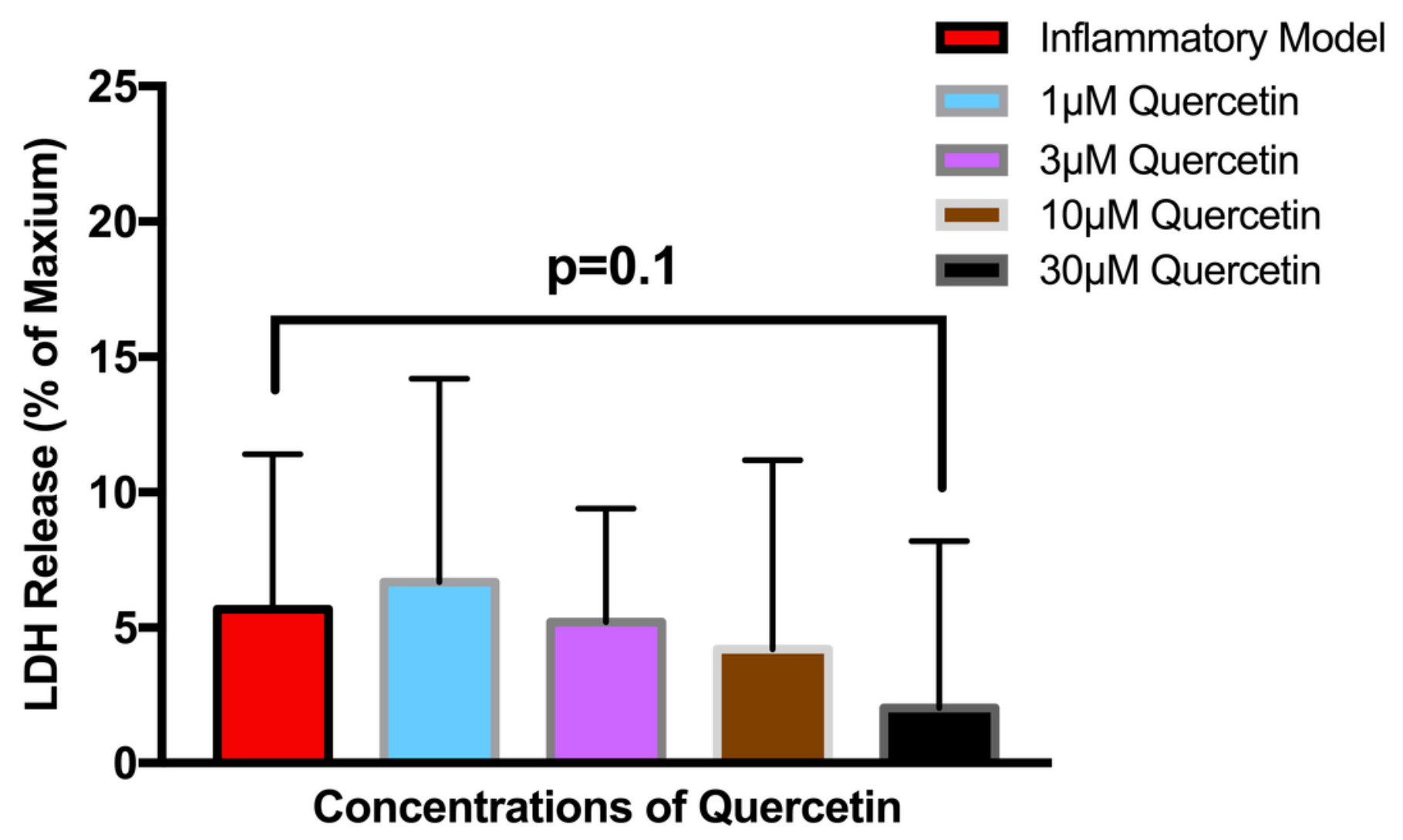


Figure 4

The effects of hydroxyurea and quercetin on LDH release.

Values are means +/- SD. The addition of $30 \mu \mathrm{mol}$ of QCT in combination with the HU treatment gleaned a significant reduction of LDH release, compared with only HU treatment.

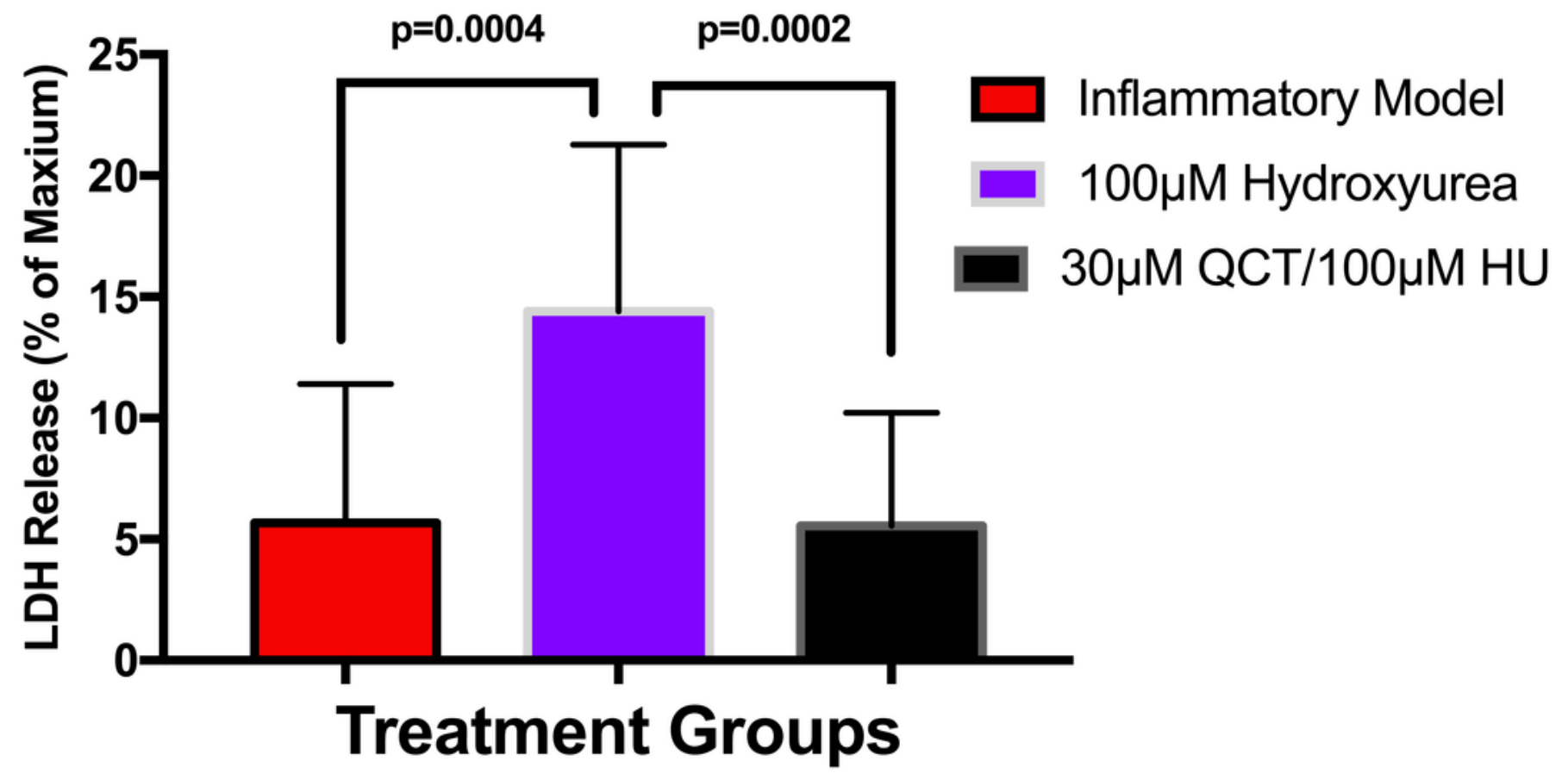

